\title{
NONLINEAR STABILITY OF A SINGLE-LAYER HYBRID GRID SHELL
}

\author{
Jianguo Cai ${ }^{1}$, Yixiang $\mathrm{Xu}^{2}$, Jian Feng ${ }^{3}$, Jin $\mathbf{Z h a n g}^{4}$ \\ ${ }^{1,3,4}$ Key Laboratory of C\&PC Structures of Ministry of Educaiton, \\ National Prestress Engineering Research Center, Southeast University, Nanjing 210096, China \\ ${ }^{2}$ Department of Civil Engineering, Strathclyde University, Glasgow, United Kingdom \\ E-mails: ${ }^{1}$ j.cai@seu.edu.cn; ${ }^{2} y i x i a n g . x u @ s t r a t h . a c . u k ;$ \\ ${ }^{3}$ seucivilfj@hotmail.com (corresponding author); ${ }^{4}$ zhangjin@seu.edu.cn
}

Received 17 Nov. 2010; accepted 25 Nov. 2011

\begin{abstract}
This paper presents a study of a hybrid grid shell, which is made of quadrangular meshes diagonally stiffened by pre-tensioned thin cables. The construction of the hybrid structure by translating a spatial curve against another spatial curve is firstly described. Then the elasto-plastic buckling analyses of the perfect hybrid structure and the corresponding single-layer lattice shell are carried out, and the influence of the asymmetric load on the failure loads is discussed based on a finite element model. Furthermore, the different shapes and sizes of imperfections are considered in this study. Two schemes of imposing imperfections are chosen: the first several eigenvalue buckling modes and the deformed shape of the loaded structure obtained from a geometrical non-linear analysis are chosen as the imperfection shape. Finally, the effects of different structural parameters, such as the rise-to-span ratio, beam section dimension, area and pre-stress of cables and boundary conditions, on the failure loads are investigated.
\end{abstract}

Keywords: hybrid structure, grid shell, stability, elasto-plastic, failure load.

\section{Introduction}

As is well known, shells still play an important role in the progress of bearing structures, particularly the singlelayer reticulated shell which carries its loads mainly by compressive forces. Therefore stability analysis is an intricate and important problem in designing reticulated shells. The single-layer reticulated shells can be regarded as a mixture of slab structure and continuum shell thus showing the stability failure of both types and even combinatorial modes (Bulenda, Knippers 2001).

The non-linear buckling analysis procedures for single-layer reticulated shells based upon non-linear finite element (FE) analysis have been developed to trace the equilibrium path by many researchers (Forman, Hutchinson 1970; Meek, Tan 1984; Borri, Spinelli 1998; Gosowski 2003; Gioncu 1995, 2003; Nie 2003). The structural behaviour of the shell structures during the whole loading process can be revealed by the load-deflection curves, by which the buckling load can be predicted. The elastic stability of the hybrid grid shell has been well studied by Bulenda and Knippers (2001). However, the analysis of elasto-plastic stability is much more complicated than the elastic analysis, since the elasto-plastic analysis involves both geometrical and material non-linearities. The elastoplastic stability of the single-layer reticulated shells has attracted more and more attentions of researchers recently (Suzuki et al. 1992; Nee, Haldar 1988; Luo 1991; Kato et al. 2000; Fan et al. 2010a, b).
Usually, triangular or quadrilateral meshes can be used in shell lattice structures. With the development of modern building industry, the appeal of glass roofs grows because of its aesthetical advantage of being translucent. Shell structures with quadrangular mesh are one of the best candidates for the transparent glass roofs. However, when opting for a quadrilateral mesh, it should be noted that the mesh is much less stiff in the plane compared to the triangular one. Moreover, the stability of shells becomes most dependent on the joint rigidity. These serious shortfalls in structural performance outweigh the apparent benefits in reducing the number and length of members used and might limit the structure to applications with short spans and low loading.

Cables are light and can provide well-defined transmission of forces. They can also been inserted into a structural system with a rational layout of members so as to make the best use of individual material properties (Hosozawa et al. 1999). To achieve high performance of the lattice shell, its quadrangular mesh should be stiffened by diagonal cables (Schlaich, Schober 1996, 1997, 1999, 2002; Schlaich 2004), as shown in Fig. 1. Note, cables under compression will not become slack when prestressed before applying external loads.

Due to its attractive mechanical properties, the hybrid grid shell has been widely used, e.g. the Word Trade Center Dresden and Atrium roof of Quartier 203 in Berlin (Schlaich, Schober 1999), the Roof of the Museum of Hamburgische Geschichte, and the glass roof of New 


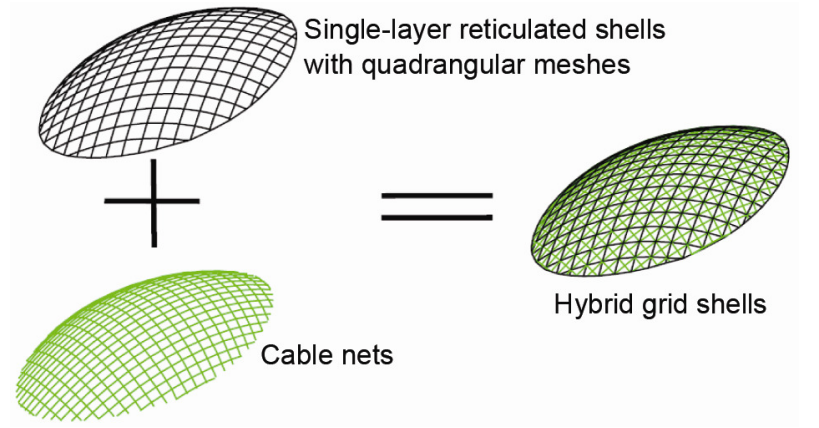

Fig. 1. The hybrid grid shell

Guangzhou Railway station in China. The geometrical non-linear finite element analysis which considers imperfections of different shapes and scales was performed to study the stability of a hybrid grid shell (Bulenda, Knippers 2001). The principle of the cable pre-stress was investigated by Schlaich (2004) based on a four-bar linkage diagonally stiffened by cables. Glymph et al. (2004) studied the constructability of a hybrid shell using planar quadrilateral glass facets for the Jerusalem Museum of Tolerance project. They described a simple but robust geometric method for achieving the structure by incorporating the necessary geometric principles into a computational parametric framework using the CATIA Version 5 system. Del Guerra and Froli (2009) proposed to roof two internal courtyards of the main building of the Engineering Faculty at the University of Pisa by means of thin flat lattice shells stiffened by a grid of steel cables. The form-finding process, construction techniques, and some case studies were concluded by Paoli (2007). He also investigated the development of high performance software and the using of new materials.

Although the structural form is used in some projects, there is little research work reported about the structural behavior of the hybrid grid shells. With the fast development of computer technology and the availability of advanced FE software, it is now possible to conduct a comprehensive study on the stability of the hybrid grid shell through geometrical and material non-linear analyses. This paper shows how to perform a geometrical and material non-linear finite element analysis by using ANSYS to investigate the stability of the hybrid grid shell. The imperfection sensitivity including the pattern and scale of imperfections will be investigated. Additionally, the effects of other factors such as the geometrical and structural parameters and the asymmetrical distribution of loads will also be taken into consideration in this paper.

\section{Design model}

Quadrangular mesh constructions require fewer machining operations on the glass and fewer mullions if the quadrilateral facets of the surface structure are maintained planar. Therefore, translation surfaces are given in Glymph et al. (2004) to guarantee the geometric planarity of facets in a quadrangular mesh system. Translating a spatial curve against another spatial curve will create a spatial surface consisting solely of planar quadrangular mesh. In this paper, to fulfill the equal length require-

ment, two identical parabolas are chosen to generate the surface. The included angle between the two planes is set to $60^{\circ}$. Finally, the surface is delimitated horizontally by intersecting a plane $\pi$ and thus gives a hybrid grid shell. The generation of the shell is illustrated in Fig. 2.

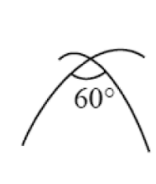

a)

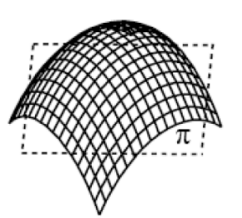

d)

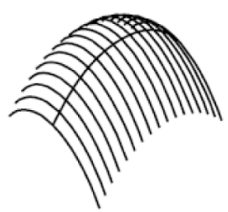

b)

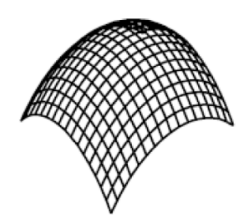

c)
Fig. 2. Generation of the surface

The design example that is used in this study is shown in Fig. 3. The major axis span and rise of the shell structure are $38 \mathrm{~m}$ and $6 \mathrm{~m}$, respectively. All nodes at the perimeter are fixed to the support. Many types of connections are commonly used in space structure construction such as welded hollow ball connections and bolt ball connections. The joints are assumed to be rigid in this study. The length of the principal single-layer lattice member is $2.0 \mathrm{~m}$, which is made of steel with a Young's modulus of $206 \mathrm{GPa}$, and the box cross-section is $60 \mathrm{~mm} \times 30 \mathrm{~mm} \times 2 \mathrm{~mm}$ (height $\times$ width $\times$ thickness). The diameter of cables is $10 \mathrm{~mm}$ and the Young's modulus is $180 \mathrm{GPa}$. The initial stress of cables is $100 \mathrm{MPa}$.

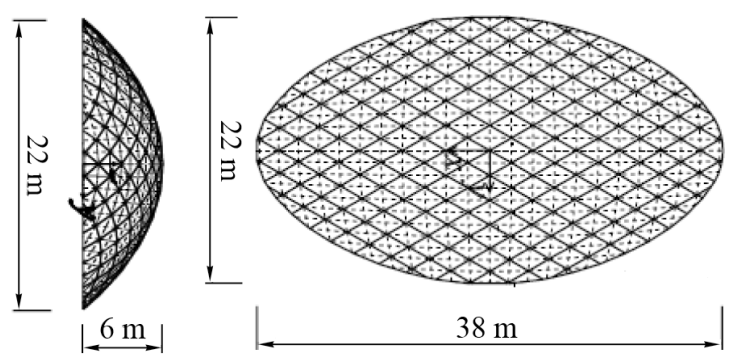

Fig. 3. The design model of the hybrid grid shell

The symmetrical load case $g+s$ (dead load +snow load) has been taken into account in all computations. The dead load $g$ consists of a self-weight of $0.5 \mathrm{kN} / \mathrm{m}^{2}$ for glass. The self-weight of all beams and cables are calculated by the software. The snow load is applied to the top surface of the structure in the vertical direction with a magnitude of $0.5 \mathrm{kN} / \mathrm{m}^{2}$. SURF 154 , which may be used for various load and surface effect applications in the software ANSYS, was used to add dead and snow loads in the vertical direction.

The Finite Element Analysis software ANSYS is employed in all structural analyses. The geometrical nonlinearity was considered by the input "NLGEOM, ON". The constitutive model of the steel was perfectly elasto- 
plastic, with the yield strength of $345 \mathrm{MPa}$. Tension-only element LINK10 is used to model cables and BEAM 189 is chosen to simulate steel beams.

\section{Stability behavior of hybrid grid shells}

The elasto-plastic stability analyses of the hybrid grid shell and the corresponding single-layer lattice shell are carried out using ANSYS. The Newton-Raphson method is used to obtain the total load-displacement equilibrium path. The load factor-displacement curves for the hybrid grid shell and the corresponding single-layer lattice shell are illustrated in Fig. 4. The load is plotted against the displacement of a node in the area of maximum deformation.

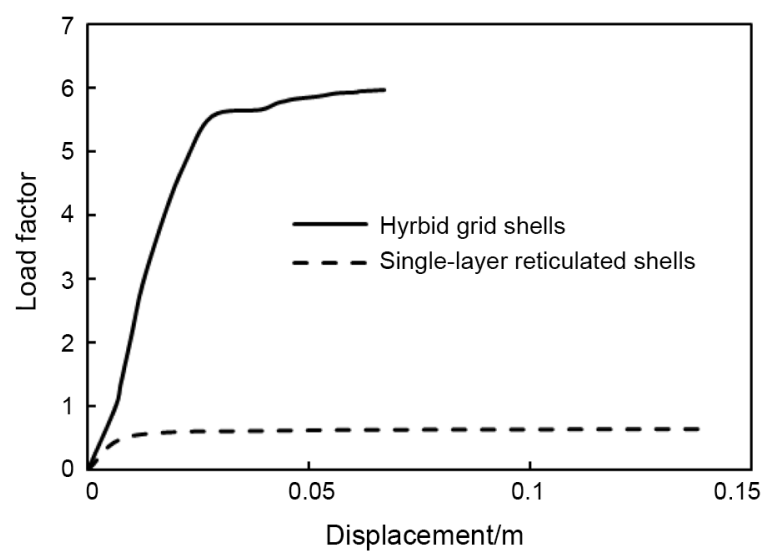

Fig. 4. Load factor vs. displacement for different structural systems

It is clear from the figure that cables do increase the stability behavior of grid shells. The buckling capacity of the hybrid grid shell is higher than that of the single-layer lattice shell. The critical buckling load factors, which are defined as the ratio of the critical buckling load to the design load given in Section 2, obtained from Fig. 4 are 5.976 and 0.636 for the two structures, respectively. It can also be found that the maximum displacement of the lattice shell is about twice that of the hybrid structure.

\section{Influence of asymmetrical loads}

Asymmetrical load case is an important factor that affects the elasto-plastic buckling capacity of hybrid grid shells. One type of asymmetrical load is the half-span load which can be resulted during construction or from snow. The asymmetrical load case $g+s / 2(g-$ dead load and $s-$ snow load, uniformly distributed over half of the span) is considered in this study. The symmetrical load case is denoted as Load 1 , and the asymmetrical load case Load 2.

Fig. 5 shows the load factor versus displacement curves for typical nodes under the asymmetrical load case. The critical buckling load factor obtained from Fig. 5 is 6.301 , which is slightly higher than that for symmetrical loads. It can be concluded that. This is because the asymmetrical distribution of load poses limited effect on the buckling load obtained from the geometrical and material non-linear analysis and the total asymmetrical loads are smaller than the symmetrical loads.

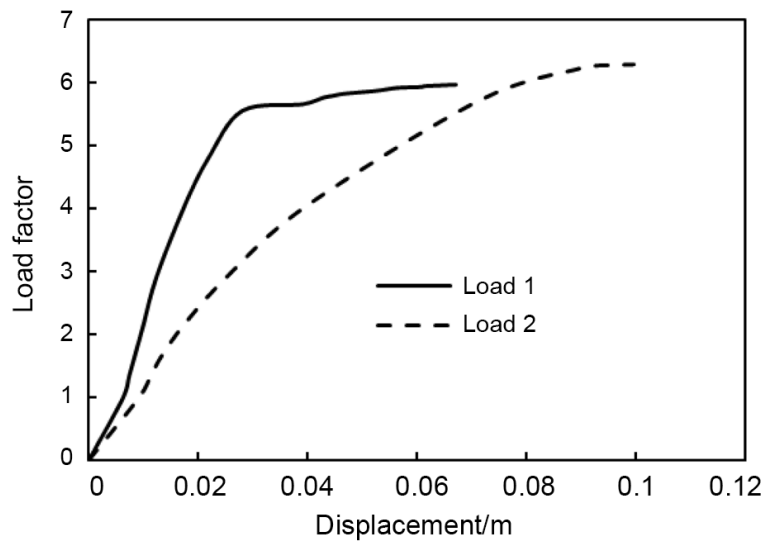

Fig. 5. Load factor vs. displacement for different loads

\section{Geometric imperfections}

Shell structures are very sensitive to geometric imperfections which are inevitable during fabrication. There are several types of imperfections in all practical structures: imperfections of the system (e.g. non-rigid joints); structural imperfections (tolerances of the cross-section area, non-homogeneous materials, etc.), loading imperfections, and geometrical imperfections (Bulenda, Knippers 2001). According to the Eurocode 3 (2007) and the Chinese code JGJ61 (2003), the geometrical imperfection should be taken into account in the non-linear analysis in order to model the structure more realistically.

Several methods are available to analyze geometrical imperfections, e.g. the random imperfection mode method (Yamada 2001), the consistent imperfection mode method (Chen, Shen 1993). In the former method, samples distributed with randomly generated imperfections are studied. The sample with the smallest buckling capacity is identified, and the corresponding buckling capacity is treated as the approximated critical capacity of the system. For the latter method, the imperfection distribution is assumed to be consistent with deflected shapes, such as eigenvalue buckling modes. The consistent imperfection mode method is used in this paper. Then the shape and the scale of the geometric imperfection will be discussed.

\subsection{Shape of the imperfection}

Normally, the first eigenvalue buckling mode is chosen as the imperfection shape. This is also called the fundamental mode imperfection method (JGJ61 2003) or the eigenmode imperfection method (EN 1993-1-6 2007). Generally, the buckling capacity that is calculated by the fundamental mode imperfection method is lower than those given by higher eigenvalue buckling modes. However, for a pre-stressed space structure, Zhang et al. (2006) stated that the buckling capacity based on other eigenvalue buckling modes may be the lowest. On the other hand, Bulenda and Knippers (2001) suggested using the final buckling shape as a geometrical imperfection.

Thus, we have set up the following shapes of imperfections for hybrid grid shells: (1) the first several eigenvalue buckling modes; (2) the displacement shape of the 
loaded structure obtained from a geometrical non-linear analysis. Both imperfections are easy to compute and therefore can be often used by engineers.

Eigenvalue buckling modes can be obtained by the eigenvalue buckling analysis. The analysis predicts the theoretical buckling capacity (the bifurcation load) of an ideal linear elastic structure. Although imperfections and material non-linearities often prevent most practical structures from achieving the theoretical elastic buckling capacity, the eigenvalue buckling analysis is still a very useful tool to estimate the critical load and buckling modes for single-layer reticulated shells. The buckling results are summarized in Fig. 6. It can be found that the asymmetrical distribution of load poses limited effect on the critical load of the hybrid structure. Some buckling modes of the structure under both loads are shown in Fig. 7. It is clear from the figure that the first three buckling modes are local modes. The instability of the hybrid structure under the asymmetric load case occurs in the half span.

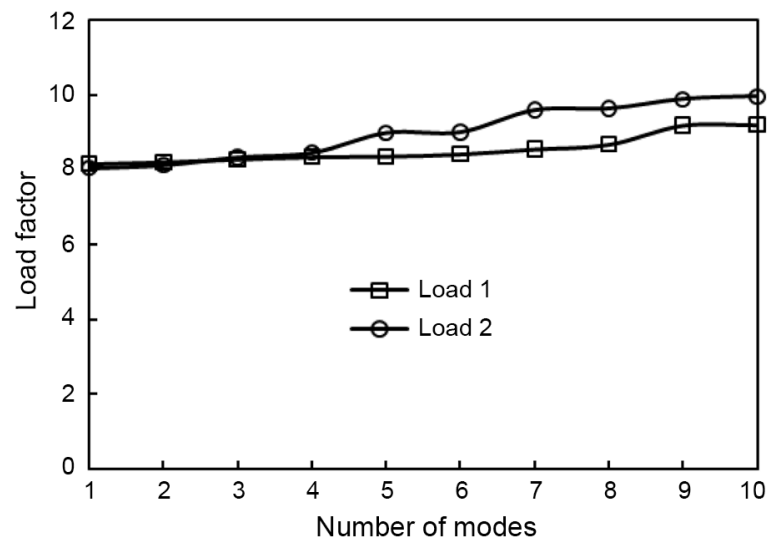

Fig. 6. Critical load factors of hybrid grid shells

For the second method of imposing imperfection, using the large deformation and elastic analysis will give deformed shapes of the hybrid grid shell under two load cases shown in Fig. 8. The deformed shapes will be then imported as imperfections in the elasto-plastic analyses.

\subsection{Scale of the imperfection}

The scaling of the imperfection is as important as its shape. Generally, the span of the structure is taken as a reference scale for the imperfection size (EN 1993-1-6 2007). According to the specifications of the Chinese lattice shell (JGJ61 2003), the maximum geometric imperfection that is caused by construction should be restricted within span $/ 300$.

As expected, the buckling capacity of the structure decreases when the maximum nodal displacement due to the geometric imperfection increases. However, for a hybrid structure, the buckling capacity when imposing an imperfection with the maximum nodal displacement of span/300 may not be the lowest (Zhang et al. 2006). Therefore, we need to vary the imperfection scale in order to assess the imperfection sensitivity of the structure.

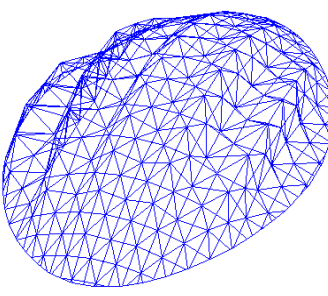

First mode

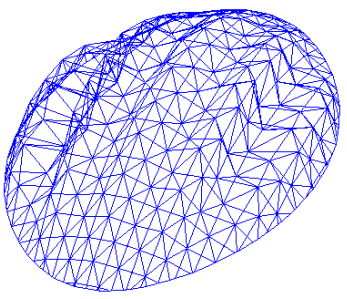

Third mode

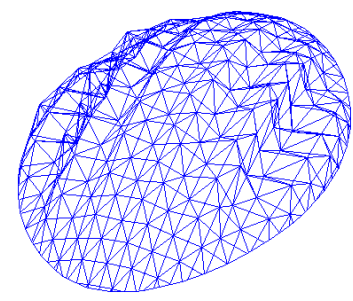

Second mode

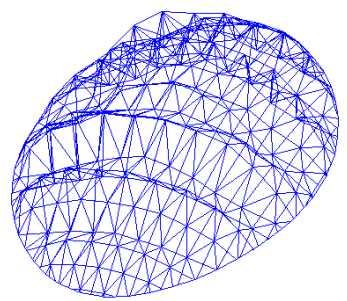

Tenth mode a) Symmetric load case

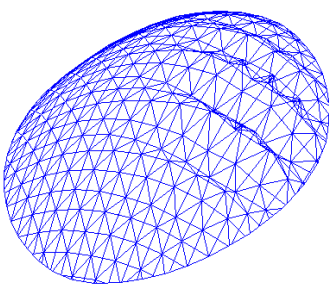

First mode

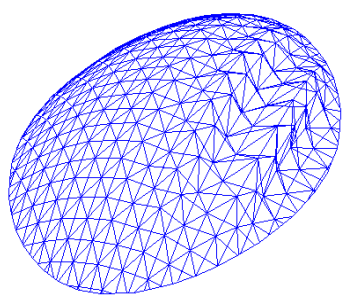

Third mode

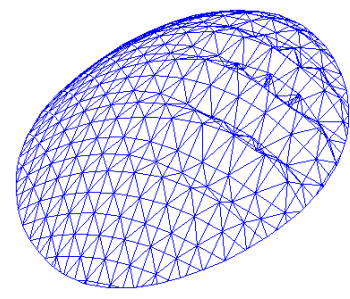

Second mode

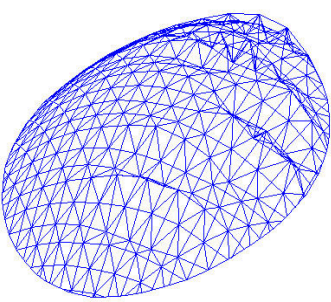

Tenth mode b) Asymmetric load case

Fig. 7. Buckling modes of hybrid structure

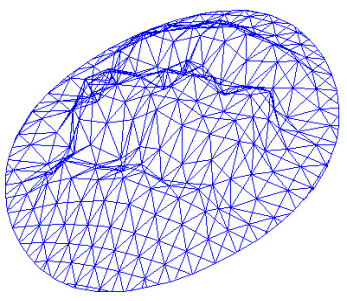

a) Symmetrical loads

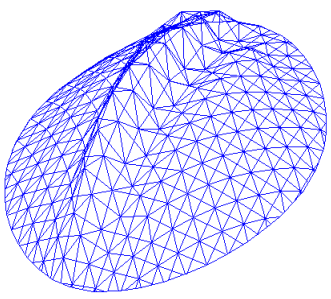

b) Asymmetrical loads
Fig. 8. Deformed shape of the hybrid grid shell in geometrical non-linear buckling 


\subsection{Results of different imperfection shapes}

For modeling of the geometrical imperfections in ANSYS package, we applied them in the form of geometric (stress-free) modifications on the model. For this reason, we obtained the imperfection model firstly and then by using the "Update Geom" order, we give values to the magnificent factor. In fact by the resulted displacement of different buckling resolution, a new model with geometrical imperfection was obtained.

Figs 9 and 10 show the load-displacement curves for different hybrid grid shells, including the perfect structure as well as the structures imposed on six different imperfect shapes. The load is plotted against a nodal displacement in the area of maximum deformation. The maximum imperfections of all shapes have been scaled to span/300. For the model in this study, the major axis span is $32 \mathrm{~m}$, and thus the maximum geometric imperfection is $106.7 \mathrm{~mm}$.

The load-displacement curves for the structure with imperfections based on eigenmodes or deformed shapes of the loading cases show the similar trend with the perfect structure. It is clear from the figure that the failure load of the hybrid structure under both loads is rapidly reduced in the presence of geometric imperfections. The

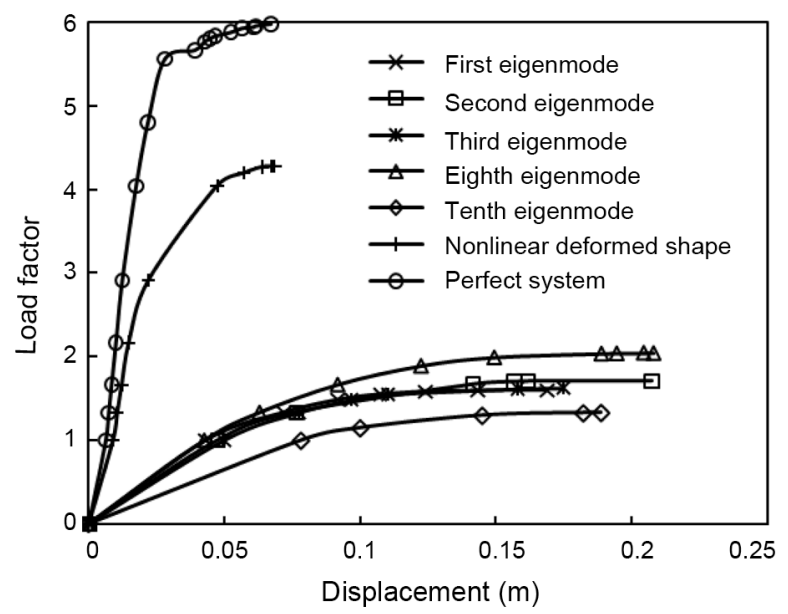

Fig. 9. Load-displacement curves for imperfect structures, loading case $g+s$

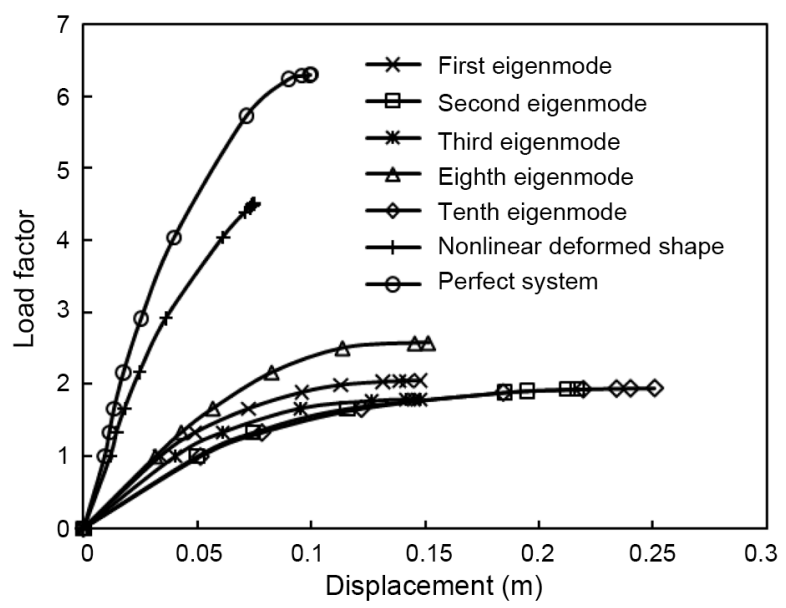

Fig. 10. Load-displacement curves for imperfect structures, loading case $g+s / 2$ lowest buckling load is predicted with the tenth eigenvalue buckling mode. In this case the failure loads are 1.334 and 1.954 times the design loads for the symmetric and asymmetric load case, respectively, yielding $77 \%$ and $70 \%$ reduction in strength. Therefore, our comparison shows large influence of different shapes of imperfections on the failure load under both loads. The tenth eigenvalue buckling mode shows the largest reduction of the failure load compared to imperfection based on other buckling shapes.

Taking into account of the complexity of implementation and the previous results, the tenth eigenmode imperfection can be straightforwardly obtained by a linearelastic eigenvalue buckling analysis and is shown as the most critical imperfection shape of the imperfection shapes considered in this paper. This imperfection shape is thus employed in all the following analyses.

\subsection{Results of different imperfection scales}

An elasto-plastic buckling analysis is carried out for the hybrid grid shell with geometric imperfection imposed. The failure loads corresponding to different scales of the tenth eigenmode are computed. Fig. 11 shows the imperfection sensitivity of the hybrid structure. It can be seen from the figure that the failure loads of the hybrid structure decrease when the positive imperfection scale increases or the negative imperfection scale decreases, i.e. when the structure is increasing its nodal deformation due to imperfection. The largest reduction rate is found around the perfect structure. Here, imposing a very small imperfection on the perfect system will lead to a big change of the failure load. Thus, the hybrid structure should be regarded as highly sensitive to imperfections. It should be noted that from the symmetry of the figure when the structure is under both load cases, the failure load reduces when the absolute value of imperfection scale increases.

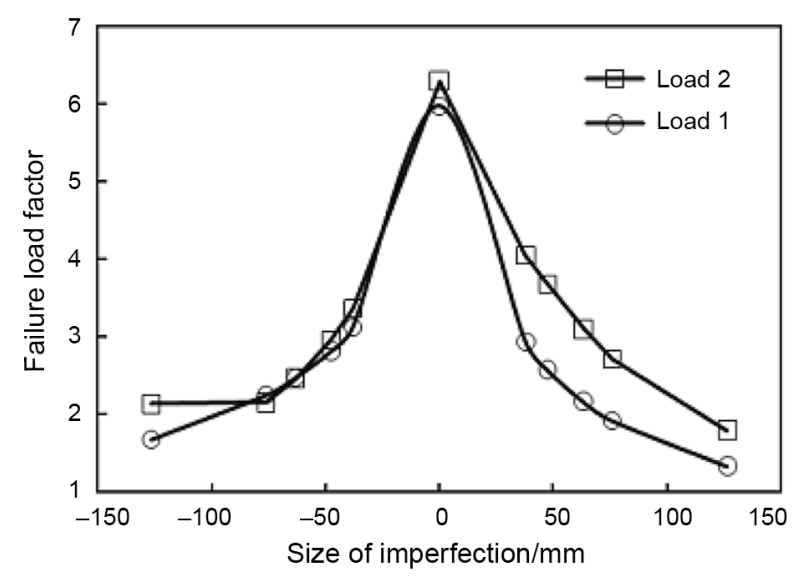

Fig. 11. Imperfection sensitivity

\section{Parametric study of the buckling capacity}

\subsection{Influence of the rise-to-span ratio}

The elasto-plastic analyses have been carried out based on sample structures with different rises (keeping the span constant). The failure loads are shown in Fig. 12. The rise-to-span ratio, which is defined as the ratio of the rise to the major axis span, corresponds to $0.16,0.22$, and 
0.30 , respectively. For the structure under the symmetrical load $g+s$, an increase in rise-to-span ratio will result in an increase in the failure load. A similar trend can be found when the structure is under the asymmetrical load $g+s / 2$. Therefore, the rise-to-span ratio is a key factor of the stability of the hybrid grid shell.

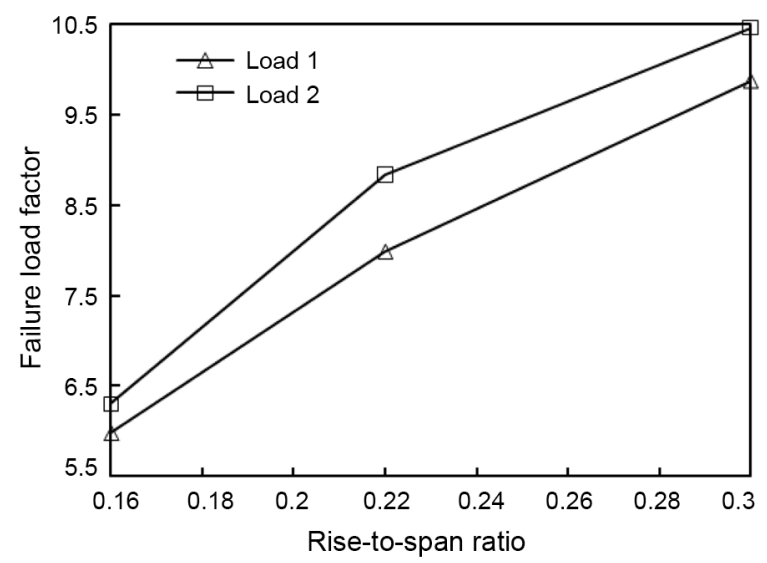

Fig. 12. Failure loads with different rise-to-span ratios

\subsection{Influence of the cross-sections of steel beams}

When the geometry of the structure is identified, the cross-section of steel beams is an important factor that affects the buckling capacity of the hybrid grid shell. Fig. 13 shows the variations of the failure load under both load cases with respect to the axial stiffness and the flexural stiffness of steel beams. It is clear from the figure that the beam section influences the failure load significantly. The failure load increases with an increase of the axial stiffness and flexural stiffness of beams. When the beam axial stiffness and flexural stiffness are reduced by about $35 \%$ and $70 \%$ from the basic model, the failure loads decrease by $55 \%$ and $45 \%$ for symmetrical and asymmetrical loads, respectively. Setting the beam axial stiffness and flexural stiffness to 2.0 and 3.5 times as those of the standard model, it doubles the failure loads for each load case, respectively. Therefore, the results show the beam section improves the stability performance of the hybrid grid shell notably.

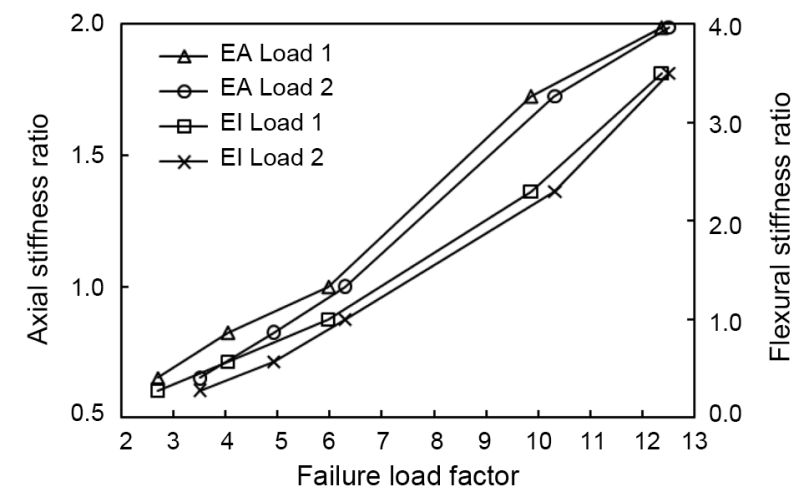

Fig. 13. Failure loads with different cross-sections of steel beams
In order to derive a relationship between the ratio of the bending and axial stiffness and the buckling behavior, the cross-sections are set to $42 \mathrm{~mm} \times 21.4 \mathrm{~mm} \times 3 \mathrm{~mm}$, $49.2 \mathrm{~mm} \times 24.6 \mathrm{~mm} \times 2.5 \mathrm{~mm}, 60 \mathrm{~mm} \times 30 \mathrm{~mm} \times 2 \mathrm{~mm}$, and $73.8 \mathrm{~mm} \times 36.9 \mathrm{~mm} \times 1.6 \mathrm{~mm}$, respectively. The axial stiffness (areas of the cross-sections) is almost the same. However, the bending stiffness is different. Fig. 14 shows the relation between the failure load and the bending/axial stiffness ratio. It is clear from the figure that the failure load increases with an increase of the ratio of the bending and axial stiffness. However, the trend becomes slow when the ratio is larger.

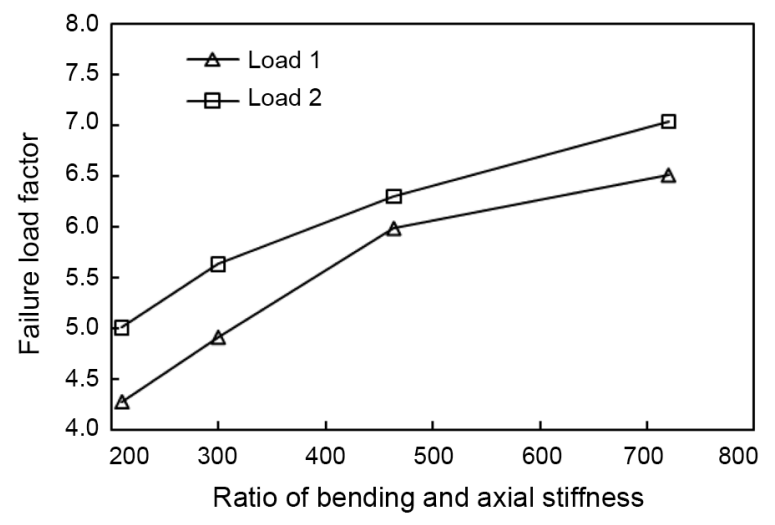

Fig. 14. Failure loads with different ratios of bending and axial stiffness

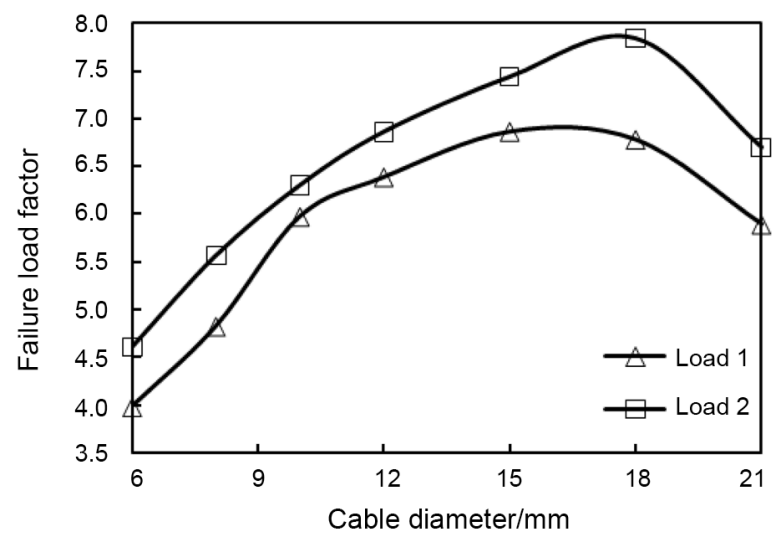

Fig. 15. Failure loads with different areas of cables

\subsection{Influence of cables}

The pre-stress and area of cables are also important factors that affect the elasto-plastic buckling behavior of the hybrid grid shell. The failure load against the diameter of cables at the specified cable pre-stress of $100 \mathrm{MPa}$ is shown in Fig. 15. The diameters of cables correspond to $6 \mathrm{~mm}, 8 \mathrm{~mm}, 10 \mathrm{~mm}, 15 \mathrm{~mm}, 18 \mathrm{~mm}$ and $21 \mathrm{~mm}$ in Fig. 15, respectively. For the structure under the symmetrical load $g+s$, if the cable diameter is smaller than $18 \mathrm{~mm}$, an increase in the cable diameter will result in an increase in the failure load. If the cable diameter is greater than $18 \mathrm{~mm}$, a higher cable area gives a lower failure load. A similar trend can be found when the structure is under asymmetrical load $g+s / 2$. Thus, it is interesting to 
note that there exits an optimal value of cable diameters for a specific hybrid grid shell.

To study the effect of different pre-stress values directly, the initial stresses are set to $50 \mathrm{MPa}, 80 \mathrm{MPa}$, $100 \mathrm{MPa}$ and $150 \mathrm{MPa}$, respectively. The failure load under both load cases versus the pre-stress of cables is shown in Fig. 16. It can be seen that the failure load increases with the increasing of the cable pre-stress. For the structure under asymmetrical loads, the differences in failure loads are very small. For the pre-stresses considered in Fig. 16, the range of the variation of failure loads is within $10 \%$. However, for the structure under symmetrical loads, when the pre-stress increase from $50 \mathrm{MPa}$ to $100 \mathrm{MPa}$, the failure load increases significantly by $55 \%$.

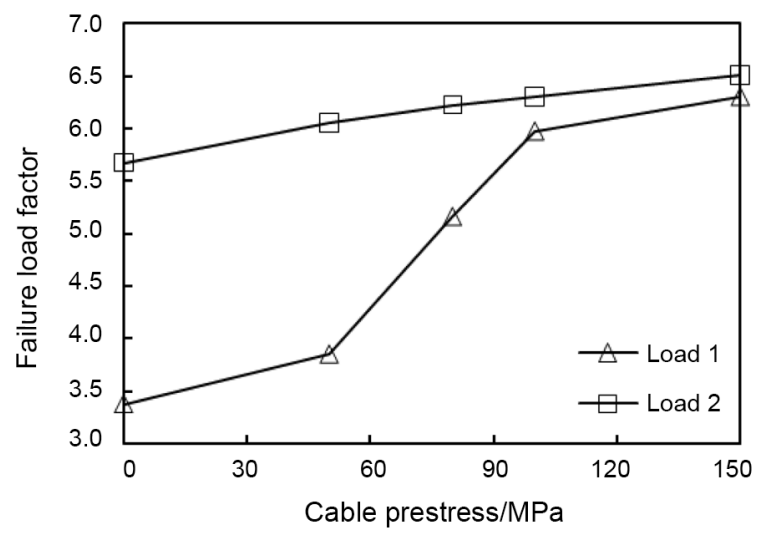

Fig. 16. Failure loads with different cable pre-stress

Therefore the influence of the cable pre-stress on the failure load is significant. It is essential to choose a proper cable diameter and prestress for a hybrid grid shell.

\subsection{Influence of boundary conditions}

In all aforementioned cases, the supports of hybrid grid shells are regarded as fixed. The elasto-plastic stability of hybrid grid shells with pinned supports is discussed in this section. The load-displacement curves with fixed and pinned supports under symmetrical and asymmetrical loads are shown in Fig. 17.

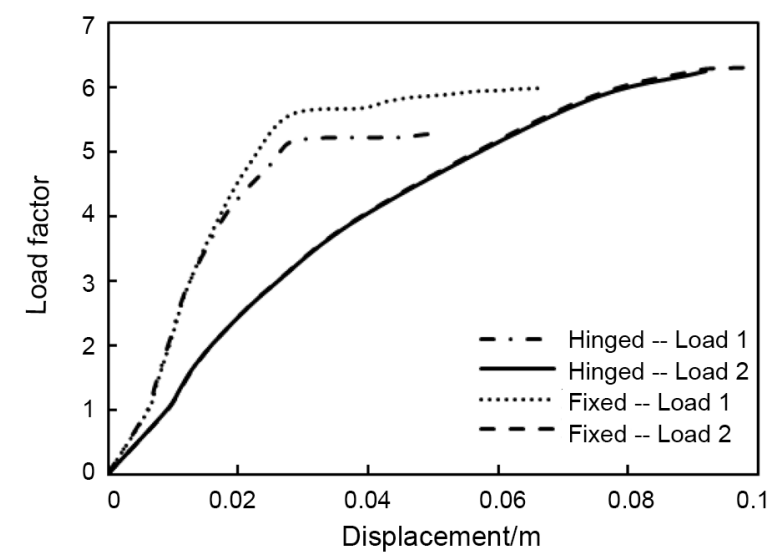

Fig. 17. Load-deflection curves of the hybrid structure with different support conditions
Fig. 17 indicates that there is a small difference of failure loads between fixed and pinned support shells. The failure load factor of the structure with pinned supports is 5.29 and 6.24 for the two load cases, respectively. Note the difference of the failure load with pinned supports between the two load cases is little. As a result, the effect of support conditions on the elasto-plastic stability is slight in the design of the hybrid grid shells.

\section{Conclusions}

The buckling capacity of the hybrid grid shell was investigated in this paper. By taking into consideration geometrical and material non-linearity using elasto-plastic analyses, the behavior of hybrid structures and the corresponding single-layer lattice shell was found significantly different. Then the effects of different geometrical, structural, and load parameters on the failure loads were studied. By comparing the results of analyses, conclusions can be drawn as follows:

1 . The effect of cables on the stability of the structure is significant. The buckling capacity of the hybrid grid shell is much higher than that of the single-layer lattice shell.

2. The stability of a hybrid grid shell is nonsensitive to the asymmetrical distribution of load.

3 . The hybrid structure is highly imperfection sensitive and the reduction of the failure load due to imperfections can be considerable. Furthermore, when imposing imperfections, the proper shape and scale of the imperfection will effectively affect the performance of the structure. The results show that the tenth eigenmode is the most critical imperfection shape to the example structure given in this paper. Furthermore, the largest reduction rate of failure load is found for the perfect structure. Thus, a very small imperfection on the perfect system will lead to a big change of the failure load.

4. The analysis results show that under a particular span, the buckling capacity increases with the rise-tospan ratio. Moreover, increasing the cross-section of steel beams notably improves the stability performance of the structure. The failure load of the hybrid grid shell initially increases with the increase of the cable diameter and then decreases afterwards. Therefore, there exists an optimal cable diameter resulting in a relatively high buckling capacity for a specific cable pre-stress. Furthermore, the influence of the cable pre-stress on the failure load is also significant. A proper cable diameter and prestress for a hybrid grid shell are required during the design.

5 . The effect of the boundary conditions on the failure load is found limited.

Demand for glass roof system with longer span is increasing, and further research is required to improve the structural form in terms of static, dynamic, and stability capacity. The above conclusions are drawn from the work of this paper that has not considered the eccentricity and semi-rigidity of joints. Further investigations addressing these details are needed in future. 


\section{Acknowledgements}

The work presented in this article was supported by the National Natural Science Foundation of China (Grant No. 50908044, 51278116), Jiangsu "Six Top Talent" Program of China (Grant No. 07-F-008), Scientific Research Foundation of Graduate School of Southeast University (Grant No. YBJJ0817), and A Project Funded by the Priority Academic Program Development of Jiangsu Higher Education Institutions. The first author would like to thank the China Scholarship Council for sponsoring his stay at the California Institute of Technology. The authors also deeply appreciate the remarks and suggestions of anonymous referees, which led to improvements in this paper.

\section{References}

Borri, C.; Spinelli, P. 1998. Buckling and post-buckling behavior of single layer reticulated shells affected by random imperfections, Computers \& Structures 30(4): 937-943. http://dx.doi.org/10.1016/0045-7949(88)90131-9

Bulenda, T.; Knippers, J. 2001. Stability of grid shells, Computers \& Structures 79(12): 1161-1174. http://dx.doi.org/10.1016/S0045-7949(01)00011-6

Chen, X.; Shen, S. Z. 1993. Complete load-deflection response and initial imperfection analysis of single-layer lattice dome, International Journal of Space Structures 8(4): 271-278.

EN 1993-1-6:2007 Eurocode 3: Design of steel structures - Part 1-6: Strength and stability of shell structures. European Committee for Standardization, 2007.

Fan, F.; Cao, Z.; Shen, S. 2010a. Elasto-plastic stability of single-layer reticulated shells, Thin-Walled Structures 48(10-11): 827-836. http://dx.doi.org/10.1016/j.tws.2010.04.004

Fan, F.; Wang, D.; Zhi, X.; Shen, S. 2010b. Failure modes of reticulated domes subjected to impact and the judgment, Thin-Walled Structures 48(2): 143-149. http://dx.doi.org/10.1016/j.tws.2009.08.005

Forman, S. E.; Hutchinson, J. W. 1970. Buckling of reticulated shell structures, International Journal of Solids and Structures 6(7): 909-932. http://dx.doi.org/10.1016/0020-7683(70)90004-1

Del Guerra, G.; Froli, M. 2009. Proposal to roof the courtyards of an historical building in Pisa with glass and steel grid shells form finding and stability problems, Journal of Architectural Engineering ASCE 15(2): 62-66. http://dx.doi.org/10.1061/(ASCE)1076-0431(2009)15: 2(62)

Glymph, J.; Shelden, D.; Ceccato, C.; Mussel, J.; Schober, H. 2004. A parametric strategy for free-form glass structures using quadrilateral planar facets, Automation in Construction 13(2): 187-202.

http://dx.doi.org/10.1016/j.autcon.2003.09.008

Gioncu, V. 1995. Buckling of reticulated shells state-of-the-art, International Journal of Space Structures 10(1): 1-46.

Gioncu, V. 2003. Stability theory: Principles and methods for design of steel structures, Journal of Constructional Steel Research 59(2): 269-270. http://dx.doi.org/10.1016/S0143-974X(02)00020-2

Gosowski, B. 2003. Spatial stability of braced thin-walled members of steel structures, Journal of Constructional Steel Research 59(7): 839-865.

http://dx.doi.org/10.1016/S0143-974X(02)00093-7
Hosozawa, O.; Shimamura, K.; Mizutani, T. 1999. The role of cables in large span spatial structures: introduction of recent space structures with cables in Japan, Engineering Structures 21(8): 795-804.

http://dx.doi.org/10.1016/S0141-0296(98)00032-7

JGJ61-2003 Technical specification for reticulated shells. Beijing: China Architecture Industry Press, 2003 [in Chinese].

Kato, S.; Yamashita, T.; Ueki, T. 2000. Evaluation of elastoplastic buckling strength of two-way grid shells using continuum analogy, in Sixth Asian Pacific conference on shell and spatial structures, October, 2000, Seoul, Korea. International Association for Shell and Spatial Structures, $105-114$.

Luo, Y. F. 1991. Elasto-plastic stability and loading completeprocess research of reticulated shells. $\mathrm{PhD}$ Thesis. Tongji University, China (in Chinese).

Meek, J. L.; Tan, H. S. 1984. Geometrically non-linear analysis of space frames by an incremental iterative technique, Computer Methods in Applied Mechanics and Engineering 47(3): 261-282. http://dx.doi.org/10.1016/0045-7825(84)90079-3

Nee, K.-M.; Haldar, A. 1988. Elastoplastic nonlinear postbuckling analysis of partially restrained space structure, Computer Methods in Applied Mechanics and Engineering 71(1): 69-97.

http://dx.doi.org/10.1016/0045-7825(88)90096-5

Nie, G. H. 2003. On the buckling of imperfect squarelyreticulated shallow spherical shells supported by elastic media, Thin-Walled Structures 41(1): 1-13. http://dx.doi.org/10.1016/S0263-8231(02)00069-1

Paoli, C. 2007. Past and future of grid shell structures. Master Thesis. Massachusetts Institute of Technology, USA.

Schlaich, J.; Schober, H. 1996. Glass-covered gird-shells, Structural Engineering International 6(2): 88-90. http://dx.doi.org/10.2749/101686696780495716

Schlaich, J.; Schober, H. 1997. Glass roof for the Hippo house at the Berlin zoo, Structural Engineering International 7(4): 252-254. http://dx.doi.org/10.2749/101686697780494581

Schlaich, J.; Schober, H. 1999. Recent glass roofs, Journal of the International Association for Shell and Spatial Structures 40: 193-205.

Schlaich, J.; Schober, H. 2002. Design principles of glass roofs, in Proc. of the International Symposium on Lightweight Structures in Civil Engineering, 24-28 June, 2002, Warsaw, Poland, 815-827.

Schlaich, J. 2004. Concept design of light structures, Journal of the International Association for Shell and Spatial Structures 45: 157-168.

Suzuki, T.; Ogawa, T.; Ikarashi, K. 1992. Elasto-plastic buckling analysis of rigidly jointed single layer reticulated domes, International Journal of Space Structures 7(4): 363368.

Yamada, S; Takeuchi, A.; Tada, Y.; Tsutsumi, K. 2001. Imperfection-sensitive overall buckling of single-layer lattice domes, Journal of Engineering Mechanics ASCE 127(4): 382-396. http://dx.doi.org/10.1061/(ASCE)0733-9399 (2001)127:4(382)

Zhang, A. L.; Zhang, X. F.; Ge, J. Q. 2006. Influence of initial geometrical imperfections on stability of a suspendome for badminton arena for 2008 Olympic Games, Spatial Structures 12(4): 8-12 (in Chinese). 
Jianguo CAI. Lecturer in School of Civil Engineering at Southeast University, Nanjing, China. He is the author of about 40 papers, published in both national and international conference proceedings and journals. His research interests focus on Long-span Structures and Deployable Structures.

Yixiang XU. Lecturer in the Departments of Civil Engineering at the University of Strathclyde, Glasgow, UK. He is the author of a number of papers, published in conference proceedings and journals. He is interested in the area of numerical study of advanced space structures as well as and design and analysis of infrastructural structures, e.g. portal frame and web crippling.

Jian FENG. Currently working as a Professor in Key Laboratory of Concrete and Prestressed Concrete Structure of Ministry of Education, Southeast University, Nanjing, China. He is the author of about 100 papers, published in both national and international conference proceedings and journals. His research interests focus on Structural Design, Prestressed Structures and Precast Structures.

Jin ZHANG. Currently working as an associate Professor in Key Laboratory of Concrete and Prestressed Concrete Structure of Ministry of Education, Southeast University, Nanjing, China. His research interests focus on Structural Design, Prestressed Structures and Timber Structures. 